\title{
A Minimally Invasive Method in Diagnosing Testicular Torsion: The Initial Experience of Scrotoscope
}

\author{
Huamao Ye, MD, ${ }^{*}$ Zhiyong Liu, MD, ${ }^{*}$ Haifeng Wang, MD, ${ }^{*}$ Yifan Chang, MD, ${ }^{*} \mathrm{Xu}$ Gao, MD, \\ Chuanliang $\mathrm{Xu}, \mathrm{MD}$, Jianguo Hou, MD, and Yinghao Sun, MD
}

\begin{abstract}
Purpose: To introduce scrotoscopy in the diagnosis of testicular torsion and evaluate its value in clinical application.

Patients and Methods: From February 2010 to June 2013, 14 patients, aged 12 to 24 years, were included into this study due to acute onset of scrotal pain. On Doppler ultrasound imaging, the blood flow decreased in seven cases (including two "no flow" cases) and remained normal in the other seven. Following anesthesia, a 10F pediatric cystoscope employed as scrotoscope was inserted into the cavity of tunica vaginalis of the testis with continued saline washing to exam the testis and epididymis.

Results: The scrotoscope had a diagnostic accuracy of 100\% (100\% specificity and 100\% sensitivity), and the color Doppler ultrasound had $77.8 \%$ specificity. Five cases were diagnosed with testicular torsion, among which four were corrected and one underwent orchiectomy. No complications were observed in these patients. Nine patients with epididymitis were given oral antibiotics, and the blood flow of the testis was normal in the testis-preserving patient. Conclusions: Our study showed that scrotoscopy could serve as a minimally invasive, safe, and effective approach in the early diagnosis of testicular torsion.
\end{abstract}

\section{Introduction}

A S A UROLOGIC EMERGENCY, testicular torsion was first described in 1840 by Delasiuave and reported in literature first by Scudder et al. ${ }^{1}$ The incidence in male under 25 years is $\sim 1$ in 4000 . $^{2}$ As a delay in diagnosis may result in loss of testicular circulation, with potential risk of infertility and reduced sperm counts, ${ }^{3}$ scrotal exploration is considered the gold standard for diagnosis and treatment. Thus, once a diagnosis has been reached, many pediatric centers in United States would be most likely to explore "acute scrotum" surgically, to avoid missed diagnosis and potential malpractice-related lawsuit. ${ }^{4}$ However, the negative surgical exploration rate is high because the symptoms often overlap with other conditions, including acute epididymo-orchitis. ${ }^{5}$ Therefore, the risk of traumatic surgery for children, who could have been treated conservatively, is very high.

To solve this problem, there may be two alternative ways. The first one is to find an alternative noninvasive examination to replace scrotal exploration without significantly increasing missed diagnosis of testicular torsion; and the other one is to invent a minimally invasive surgery to replace the open scrotal exploration. To our knowledge, the diagnosis cannot be ensured with ultrasound or other imaging techniques alone, and current diagnostic improvements, for example, implementing a clinical scoring system based upon certain clinical predictors, can only partially avoid unnecessary surgical explorations; also, Doppler ultrasound depends highly on the operator's experience. ${ }^{4}$ Moreover, novel surgical interventions that can replace scrotal exploration are yet to be invented. ${ }^{6}$ The purpose of this study is to introduce a minimally invasive method with high sensitivity and specificity in diagnosing testicular torsion.

\section{Patients and Methods}

\section{Patient and preoperative examinations}

From February 2010 to June 2013, 14 patients, aged 12 to 24 years, with acute onset of scrotum pain were admitted to

Department of Urology, Shanghai Changhai Hospital, Second Military Medical University, Shanghai, China.

*These authors contributed equally to this work.

(c) Huamao Ye et al. 2016; Published by Mary Ann Liebert, Inc. This Open Access article is distributed under the terms of the Creative Commons Attribution Noncommercial License (http://creativecommons.org/licenses/by-nc/4.0/) which permits any noncommercial use, distribution, and reproduction in any medium, provided the original author(s) and the source are credited. 
Table 1. Pain Evaluation on Postoperative Day 1

\begin{tabular}{lcccccc}
\hline & Pain with & Pain with & Pain with & Pain with & Scrotal & Scrotal \\
& NRS 0 (n) & NRS 1-3 (n) & NRS 4-6 (n) & NRS 7-10 (n) & edema (n) & hematoma (n) \\
Total \\
\hline Scrotoscopy & 0 & 8 & 1 & 0 & 0 & 0 \\
Surgical exploration & 0 & 0 & 5 & 0 & 5 & 0 \\
$p$-Value & - & 0.008 & 0.008 & - & 0.01 & 6 \\
\hline
\end{tabular}

NRS $=$ Numeric Rating Scale.

the urological center of Changhai Hospital, Shanghai, China. All patients had acute onset of pain in the hemiscrotum. No predisposing factors or fever was present in these patients. The longest time to admission was 10 hours following the onset of pain. On physical examination, the hemiscrotum was mildly to moderately congested and swollen, and the ipsilateral testis and epididymis were not palpable. All patients underwent urine and blood tests and color Doppler ultrasound. On Doppler imaging, the blood flow decreased in seven cases (including two "no flow" cases) and remained normal in the other seven cases (Table 1).

\section{Surgical procedure}

The study was approved by the institutional review board of Changhai Hospital, Shanghai, China. Before surgery, all patients were asked to sign informed consent and had full understanding of the procedure. A $10 \mathrm{~F}$ pediatric cystoscope was employed as scrotoscope. Our previous study showed that the $7 \mathrm{~F}$ semirigid ureteroscope could not be operated flexibly. General anesthesia with laryngeal mask airway or local neural blockade anesthesia was applied. Scrotoscopy was performed with the patient placed in supine position. Following skin preparation and draping, an $8 \mathrm{~mm}$ incision was made in an area with little vascularization of the scrotal skin, which was located onethird laterally and inferiorly to the hemiscrotum.

Following dissection of the scrotal skin and dartos muscle, a purse-string suture was made around the incision. Then, the parietal layer of tunica vaginalis was opened to insert the sheath of the cystoscope into the cavity of tunica vaginalis; then, the scope was introduced through the sheath. To avoid leakage around the incision, the purse-string suture was cinched, or alternatively, a pair of Allis clamps was secured at the edge of the incision (Fig. 1). Under scrotoscope, the appearance and color of the testis and epididymis and torsion of spermatic cord were examined. The scrotal contents were inspected sequentially, starting from the testis, cauda epididymis, corpus epididymis, and caput epididymis to the appendix of the testis. Continuous saline irrigation was required throughout the surgery to provide adequate space and clear surgical vision.

\section{Results}

Scrotoscopy examination was performed in all 14 patients. The general clinical characteristics of the patients were shown in Table 1. Under scrotoscope, the torsioned testis was identified, and the spermatic cord was severely twisted in five cases, indicating the presence of testicular torsion (Fig. 2). Subsequent surgical exploration confirmed the diagnosis of testicular torsion (Fig. 3). Among the five cases, four spermatic cords were rotated by $360^{\circ}$. Following relief of torsion, the testis was covered by warm and moist gauze for 15 to 20 minutes following reperfusion, and it was placed back into the scrotum and fixed in at least four sites with nonabsorbable sutures. Contralateral orchiopexy was performed simultaneously. One spermatic cord was rotated by $540^{\circ}$, and following conventional detorsion, it was still nonviable, therefore was subsequently resected. Orchiectomy of the affected testis and contralateral orchiopexy were performed in this patient. In the other nine cases, significant edema and congestion of the epididymis were identified. The diagnosis of epididymitis was subsequently confirmed. The scrotal skin was sutured with one stitch. A drainage strip was routinely placed in the hemiscrotum in all cases and removed 24 hours postoperatively.

Following the surgery, a 7-day course of oral antibiotics was given to all nine patients with epididymitis. The average time of the extra step of scrotoscopy was 29.4 (range 21-45) minutes. The first case lasted $\sim 45$ minutes. The operative time gradually decreased with accumulation of case load. And finally, the operative time stabled at about 25 minutes. For the five patients who had undertaken scrotal incision and testicular fixation, no significant complications were observed; scrotal edema was observed in all five patients at postoperative day 1 , in which three resolved after 2 weeks postoperatively, and the remaining three resolved in 3 months during follow-up. The pain measured with NRS (Numeric Rating Scale) was listed in Table 1. Color Doppler ultrasound was used for routine follow-up.

No bleeding of scrotum, testicular atrophy, or relapse of testicular torsion was documented. All 14 patients were followed for as much as 2 years (range 6-47 months). Under

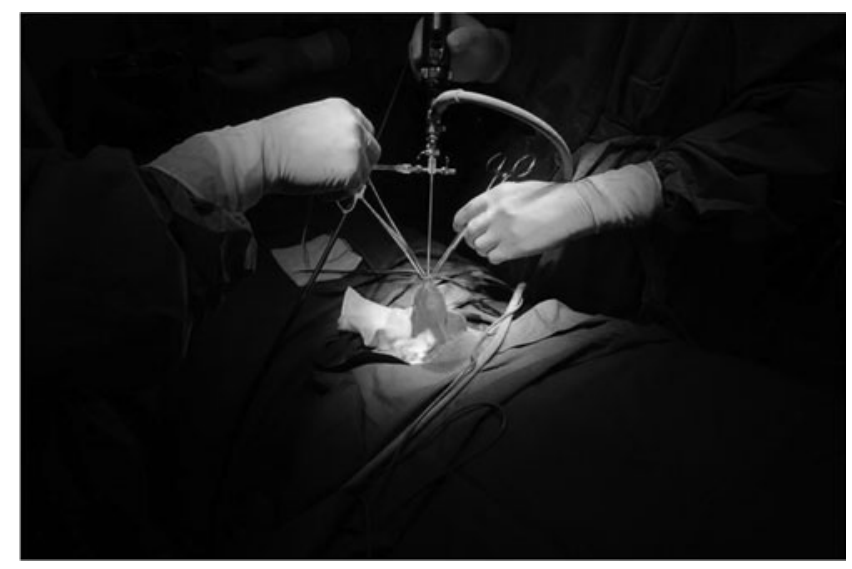

FIG. 1. A pair of Allis clamps secured at the edge of the scrotal incision to avoid leakage around the incision. 
FIG. 2. (A) An image of epididymitis present under scrotoscope (corpus epididymis). (B) A mass on the epididymis.
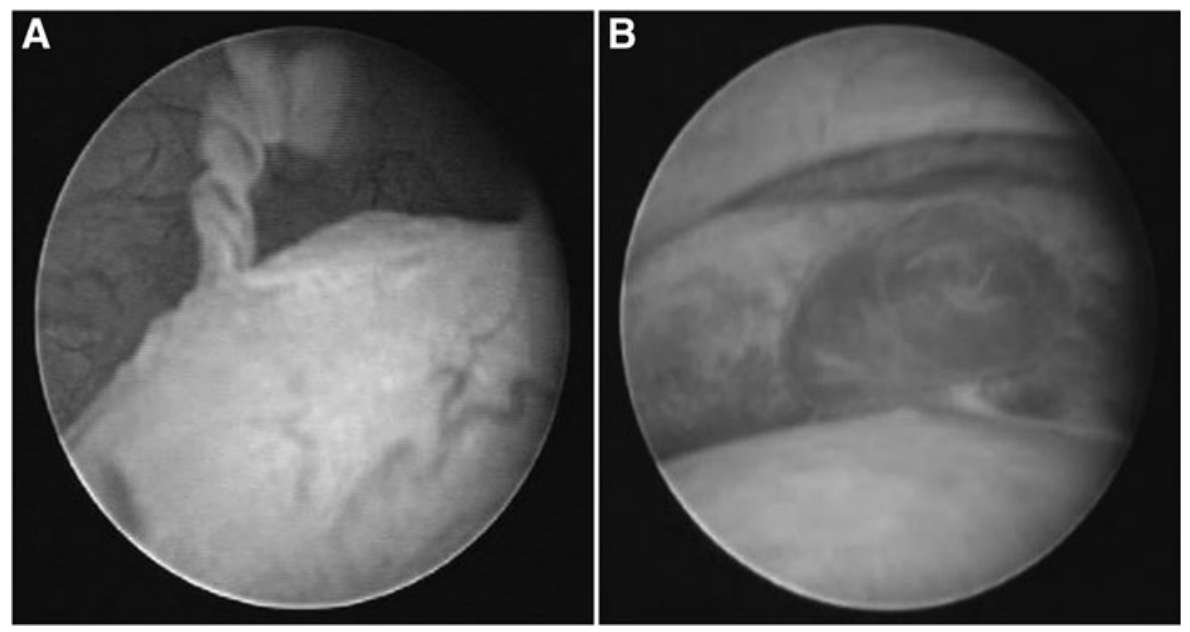

Doppler imaging, normal blood flow was seen in both testes of the testis-preserving patient.

\section{Discussion}

Testicular torsion, epididymo-orchitis, and torsion of the testicular appendix are the three most common causes of "acute scrotum" in children. ${ }^{7,8}$ Due to different management and outcomes of these three diseases, it remains an important issue to timely distinguish testicular torsion from epididymo-orchitis and torsion of the testicular appendix. Unfortunately, it is still challenging without a timely scrotal exploration. While MRI and nuclear scintigraphy have a very high detection rate, ${ }^{9,10}$ they are costly and are not universally available; more importantly, they are timeconsuming and may miss the optimal time for surgery. Similarly, Doppler ultrasound also has its advantages of excellent imaging of anatomical details and perfusion, high availability, short duration, and low costs. ${ }^{11}$ However, there are two key limitations of Doppler in the diagnosis of testicular torsion especially in infants and children, that is, the high false-negative rate and high operator dependency, ${ }^{12,13}$ especially in emergency settings. Also, our ultrasound specificity was only $71.4 \%$. Thus, many patients without testicular torsion had undergone invasive surgery. Because an ideal and noninvasive examination of testicular torsion is not available, minimizing surgical trauma becomes a more practical way to optimize the current treatment modality of testicular torsion.

Scrotoscopy is a minimally invasive surgical technique that deals with the use of endoscopy in diagnosis and treatment of the scrotal diseases. The surgery was described by Gerris and colleagues ${ }^{14}$ and Shafik. ${ }^{15}$ Gerris and colleagues employed scrotoscopy in the evaluation of infertile patients,
FIG. 3. Gross and scrotoscopic observation of testicular torsion. (A) Twisted spermatic cord; (B) necrotic epididymis; (C) necrotic testicle.

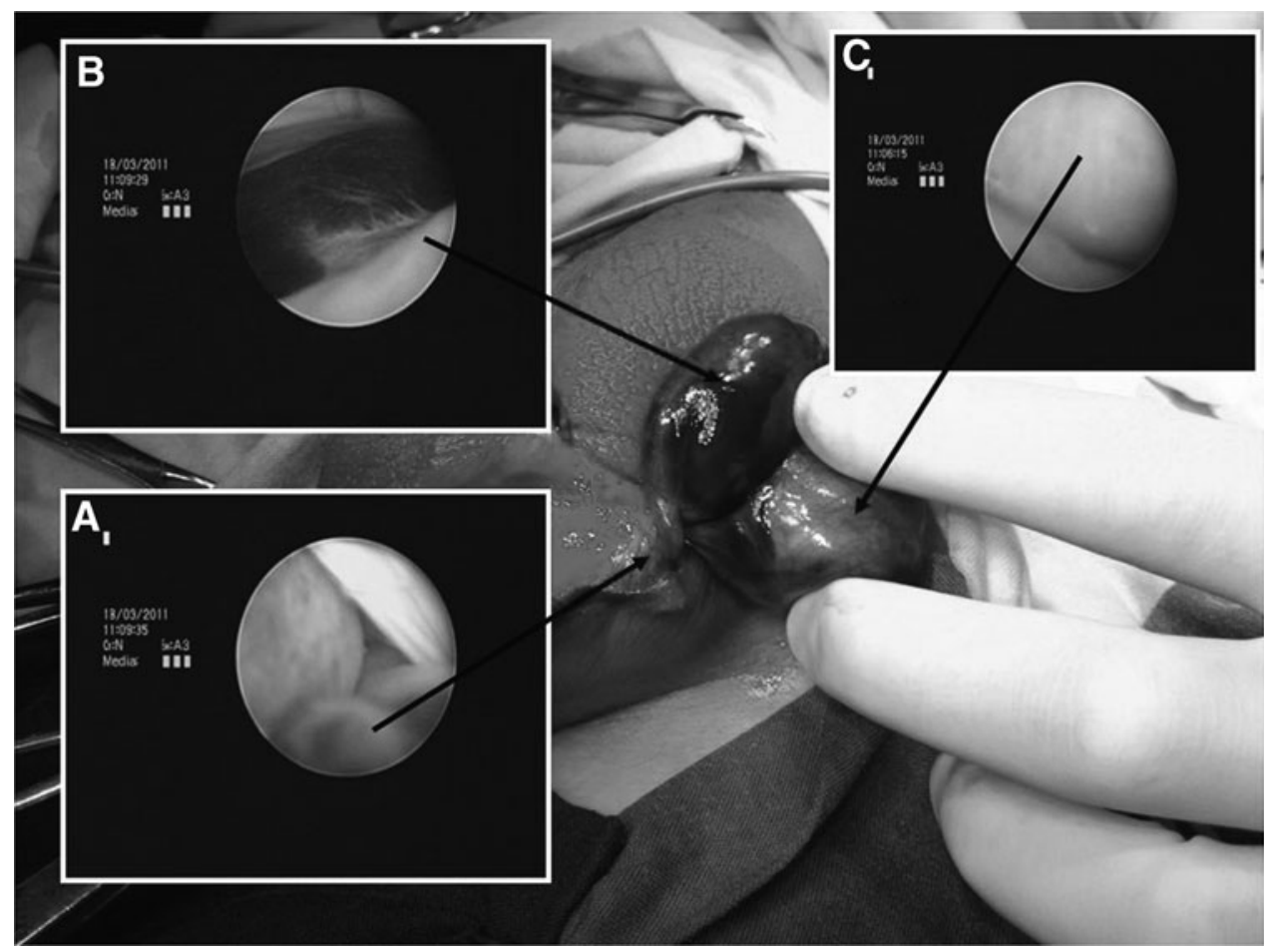


and Shafik proposed the diagnosis of questionable masses of the testis, epididymis, and spermatic cord with such technique to perform testicular biopsies in infertile patients and to perform venography and vasography. To our knowledge, the use of scrotoscope in diagnosing testicular torsion has not been reported elsewhere.

Determination of a suitable endoscope is the first important step. In the diagnosis of questionable masses in the scrotum, Shafik $^{15}$ distended the cavity of tunica vaginalis with 40 to $60 \mathrm{~mL}$ saline, creating an appropriate scrotal distention for the employment of an $18 \mathrm{~F}$ scrotoscope in the examination. However, due to swelling of the scrotal layer in patients with "acute scrotum," the cavity of tunica vaginalis cannot be well distended. Furthermore, the high pressure resulted from hydrodistention may cause further damage of the ipsilateral testis.

Initially, we adopted a $7 \mathrm{~F}$ semirigid ureteroscope in scrotoscopy examination. The semirigid ureteroscope has a diameter of 7F for the distal part, which allows easy insertion of the scope into the cavity of tunica vaginalis and is easier to navigate and observe in the space-limited cavity. However, its long shaft makes it difficult to operate precisely. Yin and colleagues ${ }^{16}$ had used adult cystoscopy. While it was easy to operate precisely, more damage would be caused. In view of this, we adopted a $10 \mathrm{~F}$ pediatric cystoscope, which has a smaller diameter compared with adult cystoscope and could enable easier navigation and more precise manipulation in the cavity with a shorter shaft.

In acute scrotum, the cavity of tunica vaginalis may not be well distended due to scrotal swelling. Thus, a limited space is created for the examination. To provide enough space for scrotoscopy examination, certain amount of hydropressure is required to distend the cavity. To achieve this goal, the incision should be as small as possible. In our study, we performed a purse-string suture around the incision. Following insertion of the scope, the purse-string suture was tied or, alternatively, a pair of Allis clamps was used to close the incision around the scrotoscope, thus enabling adequate irrigation pressure for thorough inspection of the scrotal contents.

The key point of a scrotoscopic examination is to distinguish the normal or abnormal testis and epididymis in a limited space created in the cavity of tunica vaginalis. First, the urologist should be familiar with scrotal anatomy and examine the organs consecutively. We recommend that the scrotal contents be inspected in the following order, starting from inspecting the color of the testis to the appearance of cauda epididymis, corpus epididymis, caput epididymis, and spermatic cord. Second, under scrotoscope, the color of the testis is an important factor in determining whether the testis is torsioned or not. Thus, to avoid chromatic aberration of the digital cameras, which may bring about biased color of the testis, a careful white balancing on a gauze is required before the examination. Third, during the scrotoscopy examination, the urologist should move the scope slowly with caution to avoid retracting the scope from the cavity.

Finally, the low invasiveness and less pain due to scrotoscopy examination, compared with routine exploration, enables this operation with the potential to be done under local anesthesia. As testicular torsion is a time-critical emergency, the time saved by substituting general anesthesia with local anesthesia would provide more timely treatment for the torsioned testis. Future studies with larger sample sizes should be conducted to further validate this technique. Younger child with communicating hernia might be considered a contraindication for this surgery.

\section{Conclusions}

Scrotoscopy examination is a safe and efficacious procedure, which can help in confirming the diagnosis with minimally invasive techniques. It may serve as an alternative approach against the conventional testicular exploration for reducing surgical trauma and acquiring timely intervention for acute scrotum.

\section{Acknowledgments}

This work was funded by the Major Scientific Research Proposal of the Science and Technology Commission of Shanghai Municipality (No. 09DJ1400400), General Program (No. 81272818) of the National Natural Science Foundation of China, General Program (No. 201540182) of the Health and Family Planning Commission Foundation of Shanghai, China, New Technology Foundation of Changhai Hospital, China, and Youth Startup Foundation of Changhai Hospital, China.

\section{Author Disclosure Statement}

No competing financial interests exist.

\section{References}

1. Scudder CL. II. Strangulation of the testis by torsion of the cord. A review of all recorded cases, together with the report of one recent case. Ann Surg 1901;34:234-248.

2. Sessions AE, Rabinowitz R, Hulbert WC, Goldstein MM, Mevorach RA. Testicular torsion: Direction, degree, duration and disinformation. J Urol 2003;169:663-665.

3. Mansbach JM, Forbes P, Peters C. Testicular torsion and risk factors for orchiectomy. Arch Pediatr Adolesc Med 2005;159:1167-1171.

4. Boettcher M, Krebs T, Bergholz R, Wenke K, Aronson D, Reinshagen K. Clinical and sonographic features predict testicular torsion in children: A prospective study. BJU Int 2013;112:1201-1206.

5. Boettcher M, Bergholz R, Krebs TF, Wenke K, Aronson DC. Clinical predictors of testicular torsion in children. Urology 2012;79:670-674.

6. Canpolat M, Yucel S, Sircan-Kucuksayan A, Kol A, Kazanci HO, Denkceken T. Diagnosis of testicular torsion by measuring attenuation of dual wavelengths in transmission geometry across the testis: An experimental study in a rat model. Urology 2012;79:966.e9-e12.

7. Makela E, Lahdes-Vasama T, Rajakorpi H, Wikstrom S. A 19-year review of paediatric patients with acute scrotum. Scand J Surg 2007;96:62-66.

8. Yang C, Song B, Tan J, Liu X, Wei GH. Testicular torsion in children: A 20-year retrospective study in a single institution. ScientificWorldJournal 2011;11:362-368.

9. Hod N, Maizlin Z, Strauss S, Horne T. The relative merits of Doppler sonography in the evaluation of patients with clinically and scintigraphically suspected testicular torsion. Isr Med Assoc J 2004;6:13-15.

10. Terai A, Yoshimura K, Ichioka K, Ueda N, Utsunomiya N, Kohei $\mathrm{N}$, et al. Dynamic contrast-enhanced subtraction 
magnetic resonance imaging in diagnostics of testicular torsion. Urology 2006;67:1278-1282.

11. Gunther P, Schenk JP, Wunsch R, Holland-Cunz S, Kessler $\mathrm{U}$, Troger J, et al. Acute testicular torsion in children: The role of sonography in the diagnostic workup. Eur Radiol 2006;16:2527-2532.

12. Nussbaum BAR, Bulas D, Shalaby-Rana E, Rushton G, Shao C, Majd M. Color Doppler sonography and scintigraphy of the testis: A prospective, comparative analysis in children with acute scrotal pain. Pediatr Emerg Care 2002; 18:67-71.

13. Ta A, D'Arcy FT, Hoag N, D’Arcy JP, Lawrentschuk N. Testicular torsion and the acute scrotum: Current emergency management. Eur J Emerg Med 2015 [Epub ahead of print]. DOI: 10.1097/MEJ.0000000000000303

14. Gerris J, Van Camp C, Van Neuten J, Gentens P, Van Camp K. Scrotal endoscopy in male infertility. Lancet 1988;1:1102.

15. Shafik A. The scrotoscope. A new instrument for examining the scrotal contents. Br J Urol 1990;65:209-210.

16. Yin Z, Yang JR, Wang Z, Wei YB, Yan B, Zhou KQ. Application of scrotoscope in the diagnosis and treatment of testicular and epididymal diseases [Chi]. Beijing Da Xue Xue Bao 2015;47:648-652.

Address correspondence to: Yinghao Sun, $M D$ Department of Urology Shanghai Changhai Hospital Second Military Medical University 168 Changhai Road Yangpu District Shanghai 200433 China E-mail: 446720864@qq.com

\begin{tabular}{|l|}
\multicolumn{1}{c|}{ Abbreviations Used } \\
MRI $=$ magnetic resonance imaging \\
NRS $=$ Numeric Rating Scale
\end{tabular}

